\title{
An unusual cause of gastric outlet obstruction in a young girl: Trichobezoar"
}

\author{
Mahdi Bouassida $^{1 \#}$, Hédi Charrada ${ }^{2}$, Mohamed Fadhel Chtourou ${ }^{1}$, Lamine Hamzaoui ${ }^{3}$, \\ Mohamed Monji Mighri ${ }^{1}$, Mohamed Msaddak Azzouz ${ }^{3}$, Hassen Touinsi ${ }^{1}$, Sadok Sassi ${ }^{1}$ \\ ${ }^{1}$ Department of Surgery, Mohamed Tahar Maamouri Hospital, Nabeul, Tunisia \\ ${ }^{2}$ Department of Reanimation, Mohamed Tahar Maamouri Hospital, Nabeul, Tunisia \\ ${ }^{3}$ Department of Gastrology, Mohamed Tahar Maamouri Hospital, Nabeul, Tunisia \\ Email: "bouassidamahdi@yahoo.fr
}

Received 16 August 2012; revised 22 September 2012; accepted 10 October 2012

\begin{abstract}
Trichobezoars are an infrequent form of bezoars formed from ingested hair. They are more common in adolescent females with history of trichotillomania. Exceptionally, it can occur in young children making the diagnosis difficult. We report the case of a 6 -yearold girl with a history of abdominal pain, distension, weight loss, and attacks of vomiting. Upper gastrointestinal endoscopy revealed a trichobezoar occupying almost the whole gastric cavity. The gastric bezoar was removed by surgery. Gastric trichobezoar is exceptional in young children and can lead to stunting and gastric outlet obstruction. After definitive surgical or endoscopic treatment, pediatric psychiatric consultation should be opted for any mental disorder and for prevention of further recurrence.
\end{abstract}

Keywords: Trichobezoar; Young Children; Gastric Outlet Obstruction; Trichotillomania

\section{INTRODUCTION}

Trichobezoars are collections of hair which accumulate and remain within the gastrointestinal tract for extended periods. They occur so rarely that their true incidence is unknown. Trichobezoars occur more frequently in women between the ages of 13 - 20 and often coexist with learning disabilities or psychiatric illness. These bezoars are typically found in the stomach but may also occur in the small or large bowel.

Trichobezoar have variable presentation from chronic anorexia and failure to thrive to abdominal pain, vomiting, and acute obstruction. The treatment of trichobezoar can be endoscopic or laparoscopic. If large open removal is required. This may require both gastrotomy and enterotomy if particularly extensive. It is important to refer

\footnotetext{
*Competing interests: no competing interests to declare.

\#Corresponding author.
}

for psychotherapy/psychiatric input after removal due to the risk of recurrence.

We report the case of a 6-year-old girl having a gastric outlet obstruction with stunting due to a huge gastric trichobezoard. We discuss contemporary methods in diagnosis and management of the condition.

\section{CASE REPORT}

A 6-year-old girl was referred to our department with a history of abdominal pain, distension, weight loss, and attacks of vomiting of 6 months duration. The pain increased in severity, became localized in the epigastric area and was associated with nausea and vomiting. On examination, she was $15 \mathrm{~kg}$ in weight (-2 standard deviation) and $105 \mathrm{~cm}$ in height (-2 standard deviation). There was no alopecia. Abdominal examination revealed a non-tender smooth abdominal mass in the left upper quadrant emerging from beneath the left costal margin and extending over the midline. Upper gastrointestinal endoscopy revealed a trichobezoar occupying almost the whole gastric cavity. Removal of the trichobezoar endoscopically failed as it was possible to pull only few fibers of this huge ball of hair. Laparotomy was planned and was carried out through an upper midline incision. A large $18 \times 4 \mathrm{~cm} \mathrm{~J}$-shaped foul-smelling black bunch of hairs, about $200 \mathrm{~g}$, was retrieved through a longitudinal anterior gastrotomy (Figure 1). There were no other daughter bezoars. The patient was tolerating a general diet until $5^{\text {th }}$ day after the operation and was discharged to her home on $7^{\text {th }}$ postoperative day. Psychiatric consultation was advised and child was followed up for a period of 1 year. No recurrence occurred.

\section{DISCUSSION}

Trichobezoars are an infrequent form of bezoars formed from ingested hair. The common presentation of trichobezoar is in a young female usually with an underlying 


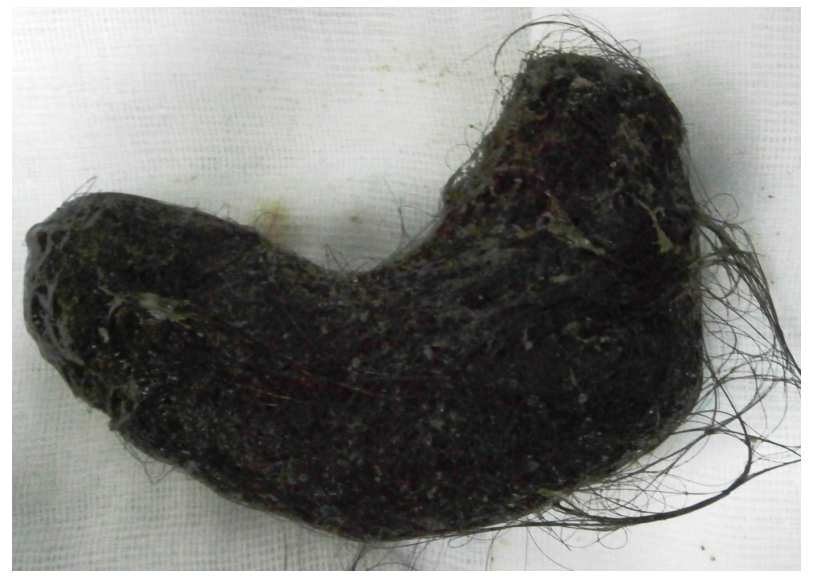

Figure 1. Stomach trichobezoar (extracted after gastrotomy).

psychiatric disorder, mainly trichophagia [1]. Ingested hair gets collected in the stomach and form a mass in the stomach and mostly these masses do not dislodge [2]. It is postulated that hair strands too slippery to be propulsed are initially retained in the mucosal folds of the stomach and become enmeshed over a period of time [3]. Trichobezoars are usually black from denaturation of protein by acid, glistening from retained mucus and foul smelling from degradation of food residue trapped within it [4]. Sometimes, these masses or part of them pass through the duodenum into the intestine and may cause ulceration, partial or total obstruction, intestinal perforation, and peritonitis [5]. Presentation of trichobezoar is usually late, due to the low index of suspicion by the clinician. A palpable abdominal mass is present in $87.7 \%$, abdominal pain in $70.2 \%$, nausea and vomiting in $64.9 \%$, weakness and weight loss in $38.1 \%$, constipation or diarrhea in 32\%, and hematemesis in 6.1\% [6]. In our case, the presentation was in a very young age; it affected the nutritional status of the child, leading to stunting, and it made the diagnosis difficult.

Ultrasonography and computed tomography (CT) scan are reliable methods for diagnosing gastrointestinal tract bezoars [7]. Recently, magnetic resonance imaging (MRI) is recommended for the evaluation of small-bowel diseases. It can be useful in determining the site and the cause of small-bowel obstructions. MRI shows the bezoar as a mass containing mottled and confluent low signal intensities on both $\mathrm{T} 1$ and $\mathrm{T} 2$ images [8].

Upper gastrointestinal endoscopy is the diagnostic procedure of choice $[9,10]$ and is also used for retrieval of proximal minor trichobezoars [9]. In the early stages, endoscopic removal is not without risk of bowel perforation and should be resolved for small trichobezoars only [2]. Surgical intervention should be reserved for patients having acute abdominal conditions or large bezoars [11]. Laparoscopic techniques are becoming popular and bezoars can be milked into the caecum before removal [2].
Open surgery is still the corner stone of large trichobezoar removal, especially if it has an extension into the bowel, which is often missed [2]. Recurrent bezoars have also been reported, especially in women and psychiatric follow-up is necessary to prevent recurrences [12,13]. A follow-up endoscopy or contrast study may also be advised if trichotillomania is suspected, as the patient usually never gives a positive history. Many of these patients report having parental discontent, bereavement, or other family problems. Parental or spouse counseling is also be advised as a regular part of treatment to prevent recurrence. The patient's long-term prognosis is excellent if behavioral therapy is used to control trichophagia, and psychological/psychiatric follow-up is maintained.

\section{CONCLUSION}

Trichobezoars occur more frequently in young women and adolescents, usually resulting from trichotillomania. It can rarely occur in children leading to a gastric outlet obstruction and stunting and making the presentation of trichobezoar late, due to the low index of suspicion by the clinician. After definitive surgical or endoscopic treatment, pediatric psychiatric consultation should be opted for any mental disorder and for prevention of further recurrence.

\section{REFERENCES}

[1] Avissar, E., Goldberg, M. and Lernau, O. (1994) Bezoar-induced ulceration and perforation of the upper gastrointestinal tract in mentally retarded patients. Pediatric Surgery International, 9, 279-280. doi:10.1007/BF00832257

[2] Jain, M., Solanki, S.L., Bhatnagar, A. and Jain, P.K. (2011) An anusual case report of Rapunzel syndrome; trichobezoard in a 3-year-old boy. International Journal of Trichology, 3, 102-104.

[3] Kawoosa, N.U. and Zargar, B.R. (2011) A giant trichobezoar causing Rapunzel syndrome in a 12-year-old female. Indian Journal of Psychological Medicine, 33, 77-79. doi:10.4103/0253-7176.85401

[4] Lamerton, A.J. (1984) Trichobezoars: Two case reportsA new physical sign. American Journal of Gastroenterology, 79, 354-356.

[5] Kovács, V., Bruncák, P., Cseri, J. and Malatinec, J. (2002) Simultaneous bezoars in the stomach, ileum and cecum causing acute ileus in a 30-year-old imbecile-case report. Rozhledy Chirurgii, 81, 248- 251.

[6] Gupta, N., Naik, S., Chaudhary, A.K., Jain, P. and Sharma, A. (2007) Rapunzel syndrome reviewed and redefined. Digestive Surgery, 24, 157-161. doi:10.1159/000102098

[7] Kaushik, N.K., Sharma, Y.P., Negi, A. and Jaswal, A. (1999) Images-gastric trichobezoar. Indian Journal of Radiology and Imaging, 9, 137-139.

[8] Lee, J.M., Jung, S.E. and Lee, K.Y. (2002) Small-bowel 
obstruction caused by phytobezoar: MR imaging findings. American Journal of Roentgenology, 179, 538-539.

[9] Shadwan, A. and Mohammad, A. (2000) Small bowel obstruction due to trichobezoar: Role of uppe endoscopy in diagnosis. Gastrointestinal Endoscopy, 52, 784-786. doi:10.1067/mge.2000.108927

[10] Billaud, Y., Pilleul, F. and Valette, P.J. (2002) Mechanical small bowel obstruction due to bezoars: Correlation between CT and surgical findings. Journal of Radiology, 83, 641-646.

[11] Mansour-Ghanaei, F., Herfatkar, M., Sedigh-Rahimabadi,
M. and Lebani-Motlagh, M. (2011) Huge simultaneous trichobezoars causing gastric and small-bowel obstruction. Journal of Research in Medical Sciences, 16, 447-452.

[12] Andrus, C.H. and Ponsky, J.L. (1988) Bezoars: Classification, pathophysiology, and treatment. American Journal of Gastroenterology, 83, 476-478.

[13] Hall, J.D. and Shami, V.M. (2006) Rapunzel's syndrome: Gastric bezoars and endoscopic management. Gastrointestinal Endoscopy Clinics of North America, 16, 111119. doi:10.1016/j.giec.2006.01.012 